\title{
Variação anual do sistema reprodutor de fêmeas de Bothrops jararaca (Serpentes, Viperidae)
}

\author{
Thélia R. F. Janeiro-Cinquini
}

Laboratório de Herpetologia, Instituto Butantan, Av. Vital Brasil, 1500, 05503-900, São Paulo, Brasil. (cinquini@butantan.gov.br)

\begin{abstract}
Annual variation of the reproductive system in females of Bothrops jararaca (Serpentes, Viperidae). The morphological changes of the ovary and oviduct of 238 tropical snakes Bothrops jararaca (Wied, 1824) were determined. The ovarian mass presented a remarkable decrease in October, after ovulation, staying in low levels from November to March, during the gestational period. From April to September, it increased because of the ovarian follicles maturation. A gradual increase in oviduct weight was observed from October to March due to stages of embryonic development. A significant difference was observed between right and left ovary weight, and oviduct length, independently of the months considered.
\end{abstract}

KEYWORDS. Bothrops jararaca, reproduction, ovary weight, oviduct length.

\section{INTRODUÇÃO}

Trabalhos sobre biologia reprodutiva dos Crotalinae neotropicais do gênero Bothrops (Wagler, 1824) são escassos e a maioria deles está baseada em informações de espécies de cativeiro (MARCH, 1928; Leloup, 1975; Melgarejo, 1977; Murphy \& Mitchell, 1984). Estudos mais recentes têm sido realizados com animais da natureza, visando complementar a biologia reprodutiva deste gênero (SAZIMA, 1992; JANEIRO-CINQUINI et al.,1993). Diversos autores (Leloup, 1973; Hoge \& Ferdersoni JR., 1976/77; LeItão-De-Araujo \& PerazZolo, 1974; Alves et al., 1988, 2000) têm estudado aspectos da biologia reprodutiva de Bothrops. FraenKel \& Martins (1940) observaram a morfologia ovariana de serpentes tropicais Xenodon merremii (Wagler, 1824), Crotalus durissus (Linnaeus, 1758), B. jararaca (Wied, 1824) e $B$. alternatus (D. B. \& D.,1854). JANEIRO-CINQUINI et al. (1993) publicaram as variações dos folículos ovarianos em $B$. jararaca durante o ano.

O ciclo reprodutivo em répteis envolve mudanças regulares e integradas em sua anatomia e fisiologia que são controladas por fatores intrínsecos (ritmo interno) ou extrínsecos (temperatura, umidade, alimento) (Moll, 1979; Fiтch, 1982). Modificações coordenadas da morfologia, fisiologia e conduta das espécies são observadas durante o ciclo reprodutivo (FITCH, 1982). Nas fêmeas, as principais mudanças morfológicas ocorrem nos ovários e ovidutos. Os ovários de répteis exibem mudanças sazonais típicas, atingindo maior tamanho na estação de reprodução, quando os folículos estão aumentados preparando-se para a ocorrência da ovulação, tornando-se menores após esta fase (MULAIK, 1946; VARMA, 1970; Presst, 1971; GoldBerg, 1975).

A morfologia dos ovidutos de poucos Squamata da região tropical tem sido estudada em detalhe (URIBE et al., 1988). Os ovidutos das serpentes apresentam múltiplas funções na fertilização, como estoque de esperma, transporte de ovos, deposição da casca, manutenção do embrião e expulsão do ovo ou feto
(Blackburn, 1998). Durante a estação reprodutiva os ovidutos também apresentam variações morfológicas, sendo maiores no período de estro (fase de vitelogênese secundária) (DESSAUER \& Fox, 1959) e menores após esta fase (Fox, 1956; Callard \& Leathem, 1970; Hoffman, 1970). Segundo Blackburn (1998), informações confiáveis sobre a morfologia do trato reprodutivo de fêmeas de Squamata são esparsas e incompletas e podem ser reconstruídas de observações fragmentadas em espécies distantemente relacionadas.

Objetiva-se analisar as alterações do estágio de desenvolvimento da massa e do comprimento dos ovários e ovidutos em B. jararaca durante o ano.

\section{MATERIAL E MÉTODOS}

O sistema reprodutivo de 238 fêmeas de $B$. jararaca provenientes das regiões sul e sudeste do Brasil foi examinado no Laboratório de Herpetologia do Instituto Butantan, de janeiro a dezembro de 1982.

Comprimento total, em $\mathrm{cm}$, comprimento rostrocloacal (CRC), em cm e massa, em g, dos espécimes foram anotados individualmente para determinar a maior e a menor fêmea com folículos vitelogênicos e ovos. O tamanho das fêmeas em relação ao tamanho dos folículos ovarianos foi observado.

Os animais sofreram eutanásia por dióxido de carbono $\left(\mathrm{CO}_{2}\right)$ e o comprimento dos ovários e ovidutos foi medido in situ por meio de laparotomia; após, os órgãos foram removidos e pesados, observando-se a presença de folículos não-vitelogênicos da classe I (menores que $0,5 \mathrm{~cm}$ de diâmetro) e II $(0,6$ a $1,0 \mathrm{~cm})$ e os vitelogênicos da classe III (1,1 a 2,0 cm) e IV (2,1 a 3,0 cm) (JaNeiro-Cinquini et al., 1993). A presença de ovos nos ovidutos, atrésicos ou em diferentes estágios de desenvolvimento embrionário, também foi verificada. Os ovários e ovidutos direito e esquerdo apresentaram variações similares durante o ano. Por esta razão, somente o ovário e oviduto direito foram utilizados na análise de massa e comprimento. Um índice foi necessário para a 
observação da variação da massa e comprimento do ovário e oviduto em relação à massa do corpo das fêmeas durante o ano: massa do ovário/massa da serpente e massa do oviduto/massa da serpente.

A análise estatística constituiu-se do teste $\mathrm{t}$ de Student com nível de significância 5\%.

\section{RESULTADOS}

As menores fêmeas de $B$. jararaca examinadas mediram $62 \mathrm{~cm}$ e as maiores $115,5 \mathrm{~cm}$ de comprimento rostro-cloacal. As fêmeas em vitelogênese (folículos classe III e IV) e com ovos, apresentaram uma variação de comprimento rostro-cloacal de 67,5 a $133 \mathrm{~cm}$ e 76,5 a $115,5 \mathrm{~cm}$ respectivamente.

As alterações morfológicas nos ovários e ovidutos de B. jararaca foram bem definidas durante o ano, devido à presença de folículos vitelogênicos e ovos. A massa do ovário apresentou uma queda de outubro a março (fig. 1). De abril a junho, houve um aumento da massa do ovário, atingindo níveis mais altos em julho, agosto e setembro, antes da ovulação, quando grandes quantidades de vitelo são acumuladas gradualmente nos folículos devido ao processo de vitelogênese. Neste período, os ovários apresentaram folículos da classe III e IV.

Foi observada variação do tamanho dos folículos ovarianos durante o ano. Folículos da classe I e II foram encontrados em todos os meses, enquanto os da classe III e IV, somente de abril a setembro (fig. 3).

Em relação ao oviduto, de abril a setembro foram encontradas somente fêmeas sem ovos (fig. 2). Em outubro, houve aumento na média da massa, permanecendo até março, período este em que foram encontradas fêmeas prenhes. De abril a setembro, ocorreu uma relação inversa da variação da massa do ovário em relação à do oviduto; foi máxima no primeiro e mínima no último (figs. 1, 2).

Uma diferença significativa entre os comprimentos

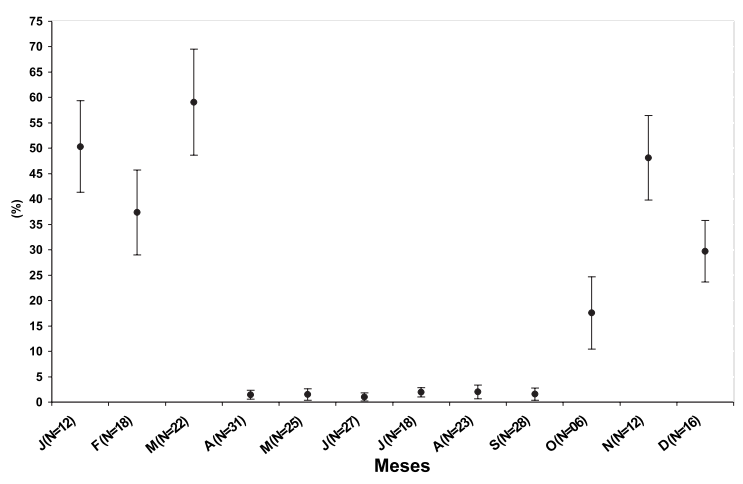

Fig. 2. Variação da média mensal da massa do oviduto/massa do corpo em Bothrops jararaca. ovário/massa do corpo em Bothrops jararaca.

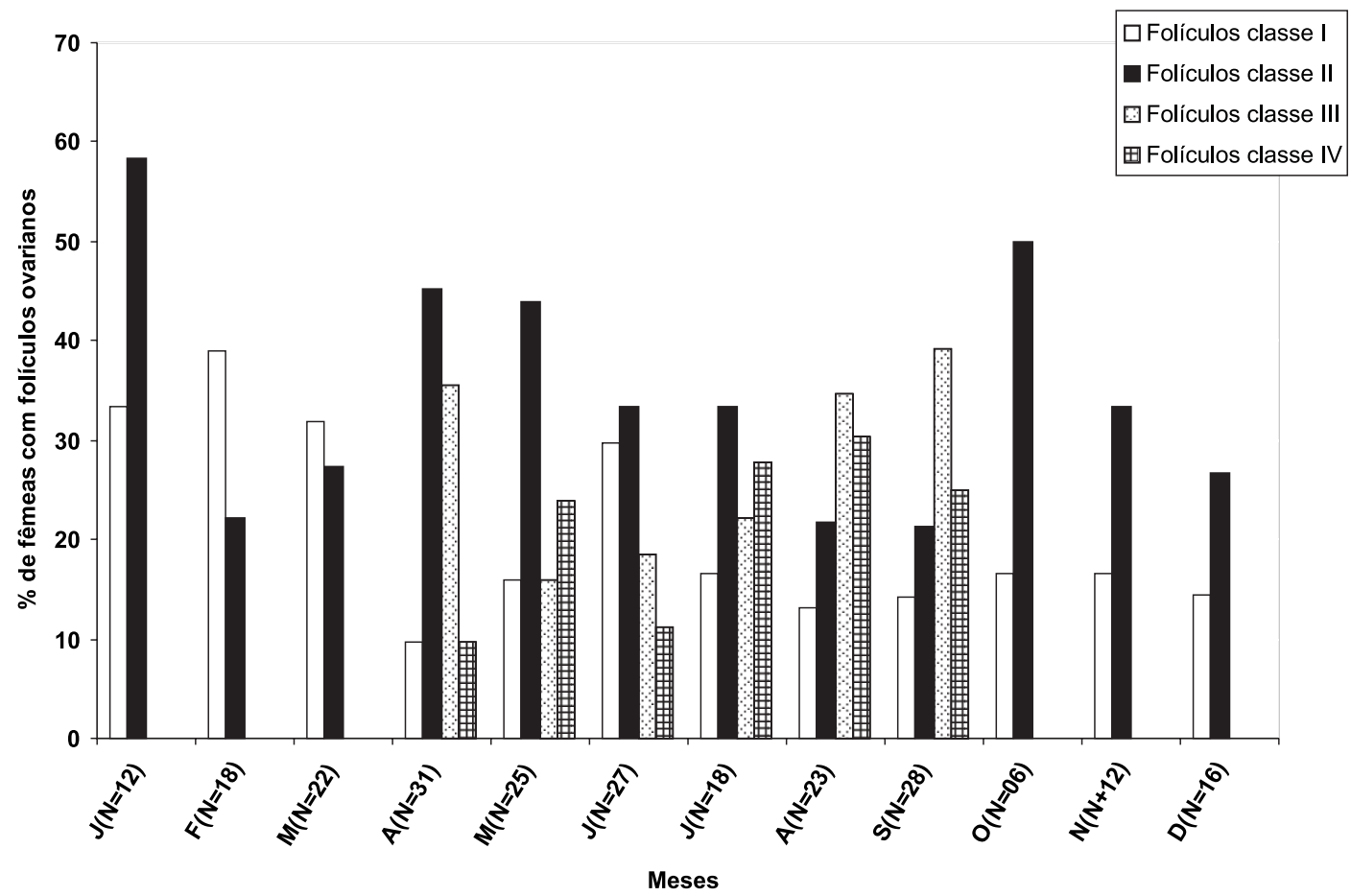

Fig. 3. Distribuição mensal da freqüência (\%) de diferentes estágios dos folículos ovarianos em fêmeas de Bothrops jararaca. 
dos ovários direito e esquerdo foi notada, sendo o direito maior que o esquerdo ( $\mathrm{t}=5,8 ; \mathrm{gl}=236 ; \mathrm{P}<0,001)$; o mesmo ocorreu com o comprimento do oviduto $(\mathrm{t}=17,13 ; \mathrm{gl}=236$; $\mathrm{P}<0,001)$.

\section{DISCUSSÃO}

Segundo SAZIMA (1992), o tamanho para maturidade em B. jararaca não é conhecido, mas foram registradas por ele fêmeas maduras com $75 \mathrm{~cm}$ e fêmeas menores prenhes com $82 \mathrm{~cm}$ de comprimento rostro-cloacal. Os dados ora analisados mostram que as fêmeas menores com folículos da classe IV apresentam comprimento rostro-cloacal de $67,5 \mathrm{~cm}$ e as prenhes $76,5 \mathrm{~cm}$, apresentando valores inferiores aos encontrados por SAZIMA (1992).

As mudanças no peso do ovário também indicaram uma relação com o crescimento folicular em Micrurus fulvius tenere (Linnaeus, 1758) (QuinN, 1979). Na serpente Naja naja (Linnaeus, 1766), foi registrado um aumento de peso dos ovários em 10 a 20 vezes no período de vitelogênese (LANCE \& LofTs, 1968; Bona-Gallo et al., 1980). Em B. jararaca, o aumento na massa do ovário foi de 12 vezes, quando comparada à média da massa de janeiro em relação a julho, indicando também uma relação direta do aumento da massa do ovário com o aumento do tamanho dos folículos ovarianos durante o processo de vitelogênese.

SAzImA (1992), estudando uma população de $B$. jararaca na natureza, e Alves et al. (1998), com B. neuwiedi (Wagler,1824) em cativeiro, observaram o comportamento de cópula nos meses de abril a junho e entre abril e julho, respectivamente. Os dados apresentados mostraram claramente o período de incidência de folículos da classe III e IV entre abril e setembro. Provavelmente neste período ocorra cópula em $B$. jararaca. Estes dados são coerentes com os dos autores acima citados, todavia, divergem em relação aos estudos de Leloup (1973), PezZAno (1986) e Alves et al. (2000), onde os períodos de cópula correspondentes são de setembro a fevereiro para B. moojeni (Hoge, 1966), fevereiro para $B$. alternatus e em fevereiro, maio, junho, agosto, outubro, novembro e dezembro, sendo mais freqüentes em outubro para B. jararaca.

Bona-GaLlo \& Licht (1983) e Licht (1984) citaram a necessidade do acasalamento em Thamnophis sirtalis parietalis (Linnaeus, 1758) como estímulo para a vitelogênese e a ovulação. Segundo Dessauer (1974), o processo de vitelogênese requer considerável quantidade de energia, utilizando progressivamente a gordura do corpo da fêmea. Sugere-se neste trabalho, cópula de abril a setembro para $B$. jararaca, pois neste período os animais se encontram em vitelogênese. A favor desta conclusão, há dois argumentos: o de economia de energia gasta no processo, pois a vitelogênese ocorreria após a fecundação e o fato de termos encontrado espermatozóides em todos os ovidutos dos animais com folículos vitelogênicos (JANEIRO-CINQUINI et al.,1993). Não seria lógico se o contrário ocorresse, pois as fêmeas teriam que procurar os machos na natureza; caso não os encontrassem, todo processo de vitelogênese e gasto de energia teriam sido inúteis.
A diminuição da massa do ovário de $B$. jararaca de outubro a março foi provavelmente devida à ovulação, pois a partir desta não foram encontrados folículos vitelogênicos IV. Estes dados assemelham-se aos apresentados por JANEIRO-CinQUiNI et al. (1993), que mostraram a variação do tamanho dos folículos ovarianos da espécie neste mesmo período.

Os ovidutos também apresentaram variações durante a estação reprodutiva. Segundo alguns autores (Evans \& ClapP, 1940; Rahn, 1942; Christiansen, 1973; KinG, 1977; Franz \& Gicca, 1982; ShanthaKumari et al., 1992), estas variações ocorreram no comprimento ou no peso do oviduto durante a vitelogênese ou durante o período gestacional nas suas três regiões: infundíbulo, útero e vagina (Giacomini, 1891; Fox, 1956; HofFman, 1970), sendo mais proeminente na região do útero (BlACKBURN, 1998).

Em fêmeas não-prenhes, o oviduto se encontrava pouco distendido e reto e sua massa foi mínima de abril a setembro. Nos animais com folículos vitelogênicos da classe III e IV, os ovidutos apresentavam-se convolutos e com câmaras de incubação para receber os folículos ovulados. Durante o período gestacional de outubro a março, houve aumento significativo da massa do oviduto devido à presença de ovos em diferentes estágios de desenvolvimento embrionário, tornando-o mais distendido e menos convoluto. Este aumento na massa do oviduto e a presença de fêmeas prenhes de outubro a março permitiram determinar o período gestacional de $B$. jararaca que é, provavelmente, em torno de 180 dias. Leloup (1973), Pezzano (1986), Solórzano \& Cerdas (1989) e Alves et al. (2000) relataram um período gestacional de 175 e 276 dias para B. moojeni, 173 dias para B. alternatus, 180 a 240 dias para B. asper (Garman, $1883)$ e 152 e 239 dias para B. jararaca. Esses dados diferem dos apresentados para B. neuwiedi, de 205 a 320 dias (Alves et al., 1998). Sazima (1992), estudando $B$. jararaca na região de Campinas, registrou as primeiras fêmeas visivelmente prenhes de outubro a novembro. Nascimentos durante a estação chuvosa foram observados por CunHA \& NASCIMENTO (1975) em B. atrox (Linnaeus, 1758) do norte do Brasil nos meses de fevereiro a março e por Leloup (1975) com B. moojeni do Brasil central em novembro e dezembro. A ocorrência de fêmeas de $B$. jararaca em estado avançado de prenhez nos meses mais quentes e úmidos do ano (novembro-dezembro) vem complementar os dados apresentados pelos autores acima citados. Segundo Seigel \& Ford (1987), a ocorrência de nascimentos durante a estação úmida pode ser uma tendência para a maioria das serpentes tropicais.

Em relação à variação interespecífica dos órgãos reprodutivos, BLACKBURN (1998) citou a variação no grau de assimetria dos ovários e ovidutos em lagartos e serpentes. Em serpentes ovíparas e vivíparas, esta assimetria é exagerada, estando o ovário direito e o útero mais anteriormente colocados do que o ovário esquerdo. Os resultados aqui obtidos com $B$. jararaca em relação ao comprimento do ovário e oviduto confirmam os observados por BLACKBURN (1998), onde o ovário direito é usualmente maior que o esquerdo. Assimetria similar também ocorreu nos ovidutos. 
Agradecimentos. Aos pesquisadores de Herpetologia do Instituto Butantan, Eliana O. Serapicos, Hana Suzuki e Radenka F. Batistic, pela leitura crítica do manuscrito e ao Sávio S. Sant'Ana pelo auxílio nos dados estatísticos.

\section{REFERÊNCIAS BIBLIOGRÁFICAS}

Alves, M. L. M.; Leitão-de-Araujo, M. \& Caberlon, E. 1998. Atividade reprodutiva de Bothrops neuwiedi em cativeiro (Serpente, Viperidae). Iheringia, Sér. Zool., Porto Alegre, (84): 185-191.

Alves, M. L. M.; Leitão-de-Araujo, M. \& Witt, A. A. 2000. Aspectos da biologia reprodutiva de Bothrops jararaca em cativeiro (Serpentes, Viperidae). Iheringia, Sér. Zool., Porto Alegre, (89):187-192.

Blackburn, D. G. 1998. Structure, function and evolution of the oviducts of squamate reptiles, with special reference to viviparity and placentation. Journal Experimental Zoology, New York, (282):560-617.

Bona-Gallo, A.; Licht, P. et al. 1980. Annual cycles in levels of pituitary and plasma gonadotropin, gonadal steroids, and thyroid activity in the Chinese cobra (Naja naja). General and Comparative Endocrinology, Orlando (42):477-493

Bona-Gallo, A. \& Licht, P. 1983. Effects of temperature on sexual receptivity and ovarian recrudescence in the garter snake Thamnophis sirtalis parietalis. Herpetologica, Chicago, (39):173-182.

Callard, I. P. \& Leathem, J. H. 1970. Biochemical changes in the oviduct of the snake Natrix sipedon pictiventris. Comparative Biochemistry Physiology, London, (32):17-22.

Christiansen, J. L. 1973. Natural and artificially induced oviducal and ovarian growth in two species of Cnemidophorus (Sauria: Teidae). Herpetologica, Chicago, (29):195-204.

Cunha, O. R. \& Nascimento, F. P. 1975. Ofídios da Amazônia VII. As serpentes peçonhentas do gênero Bothrops (jararacas) e Lachesis (surucucu) da região leste do Pará (Ophidia, Viperidae). Boletim do Museu Paraense Emílio Goeldi, Sér. Zool., Belém, (83):1-42.

Dessauer, H. C. 1974. Plasma proteins of Reptilia. In: Florkin, M. \& Scheer, B. T. eds. Chemical Zoology. New York, Academic. cap.8, p.187-216.

Dessauer, H. C. \& Fox, W. 1959. Changes in ovarian follicle composition with plasma levels of snakes during estrus. American Journal Physiology, Baltimore, (197):360-366.

Evans, L. \& Clapp, M. L. 1940. The effects of ovarian hormones and seasons on Anolis carolinensis. II. The genital system. Anatomical Record, New York, (77):57-75.

Fitch, H. S. 1982. Reproductive cycles in tropical reptiles. Occasional Papers of the Museum of Natural History University of Kansas, Lawrence, (96):1-53.

Fox, W. 1956. Seminal receptacles of snakes. Anatomical Record, New York, (124):519-540.

Fraenkel, L. \& Martins, T. 1940. Estudos sobre a fisiologia sexual das serpentes. Memórias do Instituto Butantan, São Paulo, (13):1-13.

Franz, R. \& GiCCA, D. F. 1982. Observations on the Haitian snake Antillophis parvifrons alleni. Journal of Herpetology, Kansas, (16):419-421.

Giacomini, E. 1891. Matériaux pour l'étude du development du Seps chalcides. Archives Italiennes de Biologie, Pisa, (16):332-359.

GoldBERG, S. R. 1975. Reproduction in the sagebrush lizard, Sceloporus graciosus. American Midland Naturalist, Notre Dame, (93):177-187.

Hoffman, L. H. 1970. Placentation in the garter snake, Thamnophis sirtalis. Journal of Morphology, New York, (131):57-88.

Hoge, A. R. \& Federsoni, P. A., JR. 1976/1977. Observações sobre uma ninhada de Bothrops atrox (Linnaeus, 1758) [Serpentes: Viperidae: Crotalinae]. Memórias do Instituto Butantan, São Paulo, (40/41):19-36.
Janeiro-Cinquini, T. R. F.; Leinz, F. F. et al. 1993. Ovarian cycle of the snake Bothrops jararaca. Memórias do Instituto Butantan, São Paulo, 55(1):33-36.

KING, M. A. 1977. Reproduction in the Australian gekko Phyllodactylus marmoratus (Gray). Herpetologica, Chicago, (33): 7-13.

Lance, V. \& Lofts, B. 1968. Studies on the annual reproductive cycle of the female cobra, Naja naja. Journal of Morphology, New York, (157):161-180.

Leitão-de-Araujo, M. \& Perazzolo, M. 1974. Nota prévia sobre a biologia de tanatofídios em cativeiro (Ophidia, Elapidae e Viperidae). Iheringia, Sér. Zool., Porto Alegre, (45):55-66.

Leloup, P. 1973. Essais de rationalisation dans le maintien d'un serpentarium à but industruel. Acta Tropica, New Castle, 30(4):281-311.

1975. Observations sur la reproduction de Bothrops moojeni (Hoge 1966) en captivité. Acta Zoologica et Pathologica Antverpiensia, Antwerpen, (78):177-198.

Licht, P. 1984. Reptiles. In: Lamming, G. E. ed. Marshalls physiology of reproduction. Edenburgh, Churchill Livingston, v.1, p.206-282.

MARCh, D. D. H. 1928. Field notes on barba amarilla (Bothrops atrox). Bulletin Antivenin Institute of America, Philadelphia, (1):92-97.

Melgarejo, A. 1977. Observaciones sobre nacimiento en el laboratorio de Bothrops neuwiedi pubescens (Cope, 1870) (Ophidia, Crotalinae). Revista de Biologia del Uruguay, Montevideo, (5):35-41.

Moll, E. O. 1979. Reproductive cycles and adaptations. In: Harless, M. \& Morlock H. eds. Turtles: Perspectives and Research. New York, John Wiley \& Sons. p. 305-331.

Mulaik, D. 1946. A comparative study of the urogenital systems of an oviparous and two ovoviviparous species of the lizard genus Sceloporus. Bulletin University Utah Biology Series, Utah, (37):3-24

Murphy, J. B. \& Mitchell, L. A. 1984. Miscellaneous notes on the reproductive biology of reptiles. 6. Thirteen varieties of the genus Bothrops (Serpentes, Crotalinae). Acta Zoologica et Pathologica Antverpiensia, Antwerpen, (78):199-214.

PezZANo, V. 1986. Reproduction of Bothrops alternatus (Dumèril, Bibron \& Dúmeril, 1854) in captivity. Litteratura Serpentiun, Asten, 6(1):13-18.

Presst, I. 1971. An ecological study of the viper Vipera berus in southern Britain. Journal of Zoology, London, (164):373418 .

QuinN, H. R. 1979. Reproduction and growth of the Texas coral snake (Micrurus fulvius tenere). Copeia, Lawrence, 1979(3): $453-463$.

RAHN, H. 1942. The reproductive cycle of the praire rattler. Copeia, Lawrence, 1942:233-240.

SAzima, I. 1992. Natural history of the jararaca pitviper, Bothrops jararaca, in southeastern Brazil. In: CAmpBell, J. A. \&. Brodie, E. D., JR. eds. Biology of the Pitvipers, Texas, Selva Tyler. p.199-216.

SEIGEL, R. A. \& Ford, N. B. 1987. Reproductive ecology. In: Seigel, R. A.; Collins, J. T. \& Novak, S. S. eds. Snakes: Ecology and Evolutionary Biology. New York, MacMillan. p. 210-252.

Shanthakumari, T. R.; Sarkar, H. B. D. et al. 1992. Histological, histochemical, and biochemical changes in the annual oviduct cycle of the agamid, Calotes versicolor. Journal of Morphology, New York, (211):295-306.

Solórzano, A. \& Cerdas, L. 1989. Reproductive biology and distribution of the terciopelo, Bothrops asper Garman (Serpentes: Viperidae) in Costa Rica. Herpetologica, Chicago, 45(4):444-450.

Uribe, M. C. A.; Velasco, S. R. et al. 1988. Oviduct histology of the lizard, Ctenosaura pectinata. Copeia, Lawrence, 1988(4): 1035-1042.

VARmA, S. K. 1970. Morphology of ovarian changes in the garden lizard Calotes versicolor. Journal of Morphology, New York, (131):95-209.

Recebido em maio de 2003. Aceito em abril de 2004. ISSN 0073-4721 